\title{
Harmonic Detection and Control of Power System Based on Improved
}

\section{EEMD Algorithm}

\author{
Lei Chen ${ }^{1 a}, \quad$ Guochu Chen ${ }^{1, a}$ \\ ${ }^{1}$ School of Electric Engineering, Shanghai DianJi University, 200240 Shanghai , China \\ aCCorresponding author:chengc@sdju.edu.cn
}

Keywords: Harmonic Detection; Power System; EEMD Algorithm

\begin{abstract}
Combined with the traditional Empirical Mode Decomposition(EMD),Ensemble Empirical Mode Decomposition(EEMD) and Complementary Ensemble Empirical Mode Decomposition(CEEMD) method, in view of the modal confusing problems, proposed an improved algorithm based on EEMD, and expounds the calculation and realization process, through simulation experiment, the results show that the improved method can find the mixed wave of fundamental wave, very suitable for power system harmonic detection and governance.
\end{abstract}

\section{Introduction}

Since the formation of electric power system, the harmonic of power system has been a problem that need to be solved.In the process of detecting harmonics, the low pass filter must be used to extract the fundamental wave and to make a difference to the original signal(such as instantaneous reactive power theory(p-q algorithm)、 $\mathrm{i}_{\mathrm{p}} \mathrm{i}_{\mathrm{q}}$ algorithm and frequency tracking algorithm $\left.{ }^{[1]}\right)$. This leads to a direct relationship between the speed of the dynamic tracking and the performance of the low pass filter when the harmonic detection is carried out.

Based on common method of harmonic detection and governance necessary prerequisite basis, in combination with EMD、EEMD、CEEMD, for modal confusing problems, this paper proposes a new based on improved EEMD power system harmonic detection and governance algorithm, The algorithm has no requirement on the original current or voltage signal, and does not need the synchronous angle of the grid, and can be used to detect the fundamental and harmonic in single-phase, three-phase three wire or three phase four wire system.

\section{Basic Theory}

$\mathrm{EMD}^{[2,3]}$ is a data driven signal processing method proposed by Huang etc. ${ }^{[4,5]}$ in late twentieth Century, and has been applied in many fields.However, there are also many problems in this method, the main problem is the mode confusion ${ }^{[2,6]}$. The so-called modal confusion refers to:In the same IMF, there are many different scales or different frequency signals, Or the signal is decomposed into multiple IMF components with the same scale or the same frequency ${ }^{[2,7]}$.

Wu etc. ${ }^{[8]}$ proposed EEMD, this method in the original signal in many times to join different white noise EMD decomposition, the results of the decomposition and the average, get IMF.However, if the white noise amplitude and the number of iterations are not appropriate, not only will increase the amount of computation, but also the IMF will appear a lot of false components, not only so that it also needs to continue to deal with the results. Yeh etc. ${ }^{[9]}$ proposed 
CEEMD, to add two sets of equal size to the original signal in the opposite direction as the white noise signal, The decomposition effect of this method is equivalent to that of EEMD, but to some extent, the reconstruction error caused by white noise is reduced.

\section{Improved EEMD Theory}

Improved EEMD Theory On the basis of the research of $\mathrm{Wu}$ etc. ${ }^{[14]}$ Yeh etc. ${ }^{[15]}$, proposed an improved EEMD method,this method can be used to filter the high frequency part of the signal, and find the fundamental wave in the mixed wave, which can be summarized into the following steps.

1)The original signal $S(t)$, added in modulus value equal to the positive and negative two groups of white noise signal $\mathrm{w}(\mathrm{t})$ and $-\mathrm{w}(\mathrm{t})$.

as below:

$$
\begin{aligned}
& \mathrm{S}_{1}(\mathrm{t})=\mathrm{S}(\mathrm{t})+\mathrm{w}(\mathrm{t}) ; \\
& \mathrm{S}_{2}(\mathrm{t})=\mathrm{S}(\mathrm{t})-\mathrm{w}(\mathrm{t}) ;
\end{aligned}
$$

the standard deviation between $\mathrm{w}(\mathrm{t})$ and the original signal is 0.2 。

2)Respectively to A, B the EMD decomposition.

$$
\mathrm{S}_{1}(\mathrm{t}) \stackrel{E M D}{\longrightarrow} \mathrm{C}_{\mathrm{i}+}(\mathrm{t})
$$

$$
\mathrm{S}_{2}(\mathrm{t}) \stackrel{E M D}{\longrightarrow} \mathrm{C}_{\mathrm{i}-}(\mathrm{t}) \text {; }
$$

The $\mathrm{C}_{\mathrm{i}+}(\mathrm{t})$ and $\mathrm{C}_{\mathrm{i}-}(\mathrm{t})$ in the formula represent the decomposition results of the two groups respectively.

3 ) Add different white noise repeat step 2), 1) $\mathrm{N}$ times( $\mathrm{N}$ take 200), and carry on the set total average.

$$
\begin{aligned}
& \mathrm{X}_{1}(\mathrm{t})=\frac{1}{N} \sum_{i=1}^{N} C_{\mathrm{i}+}(t) ; \\
& \mathrm{X}_{2}(\mathrm{t})=\frac{1}{N} \sum_{i=1}^{N} C_{\mathrm{i}-}(t) ;
\end{aligned}
$$

then $\mathrm{S}(\mathrm{t})=\frac{1}{2}\left(\mathrm{X}_{1}(\mathrm{t})+\mathrm{X}_{2}(\mathrm{t})\right)=\sum_{\mathrm{r}=1}^{m} C_{r}(t)+R(t)$

$\mathrm{C}_{\mathrm{r}}(\mathrm{t})$ is IMF component, $\mathrm{R}(\mathrm{T})$ is the margin.

4) Because of the IMF component of the EMD after the decomposition has serious mode confusion, and the component is from high frequency to low frequency output, so $\mathrm{C} 1(\mathrm{t})$, $\mathrm{C}_{2}(\mathrm{t}) \mathrm{L} \mathrm{C}_{\mathrm{m}}(\mathrm{t}), \mathrm{R}(\mathrm{t})$ for the following decomposition:

$$
\begin{aligned}
& \mathrm{C}_{1}(\mathrm{t}) \text { as the original signal } \stackrel{\text { step 1),2),3) operation }}{\longrightarrow} \mathrm{b}_{1}(\mathrm{t})+\mathrm{B}_{1}(\mathrm{t}) \text {; } \\
& \mathrm{C}_{2}(\mathrm{t})+\mathrm{B} 1(\mathrm{t}) \text { as the original signal } \stackrel{\text { step } 1), 2), 3) \text { operation }}{\longrightarrow} \mathrm{b}_{2}(\mathrm{t})+\mathrm{B}_{2}(\mathrm{t}) \text {; } \\
& \mathrm{C}_{\mathrm{m}}(\mathrm{t})+\mathrm{B}_{\mathrm{m}-1}(\mathrm{t}) \text { as the original signal } \stackrel{\text { step } 1), 2), 3) \text { operation }}{\longrightarrow} \mathrm{b}_{\mathrm{m}}(\mathrm{t})+\mathrm{B}_{\mathrm{m}}(\mathrm{t}) \text {; }
\end{aligned}
$$




$$
\mathrm{R}(\mathrm{t})+\mathrm{B}_{\mathrm{m}}(\mathrm{t}) \text { as the original signal } \stackrel{\text { step 1),2),3) operation }}{\longrightarrow} \mathrm{b}_{\mathrm{m}+1}(\mathrm{t})+\mathrm{B}_{\mathrm{m}+1}(\mathrm{t}) \text {; }
$$

$b_{1}(t), b_{2}(t), \ldots b_{m+1}(t)$ are first decomposed IMF component, respectively.

then $S(t)=\sum_{i=1}^{m+1} b_{i}+B_{m+1}(t)$

$$
r(t)=S(t)-B_{m+1}(t)
$$

$B_{m+1}(t)$ is the remaining fundamental wave,

$\mathrm{r}(\mathrm{t})$ is the total harmonic.

Simulation Experiment and Result Analysis In order to test the validity of the proposed method, the simulation experiment of the signal $x(t)$ is carried out:

$$
x(t)=a_{1}(t)+a_{2}(t)+b(t)
$$

among them, $\mathrm{t}=1: 1000 ; \mathrm{a}_{1}(\mathrm{t})=\sin (\mathrm{t} / 100 * 2 * \mathrm{pi}) ; \mathrm{a}_{2}(\mathrm{t})=0.2 \sin (10 \mathrm{t} / 100 * 2 * \mathrm{pi}) ; \mathrm{b}(\mathrm{t})$ is the two stage intermittent signal, the amplitude is 0.5 .

Mixed signal $\mathrm{x}(\mathrm{t})$ and its components are shown in figure 1.

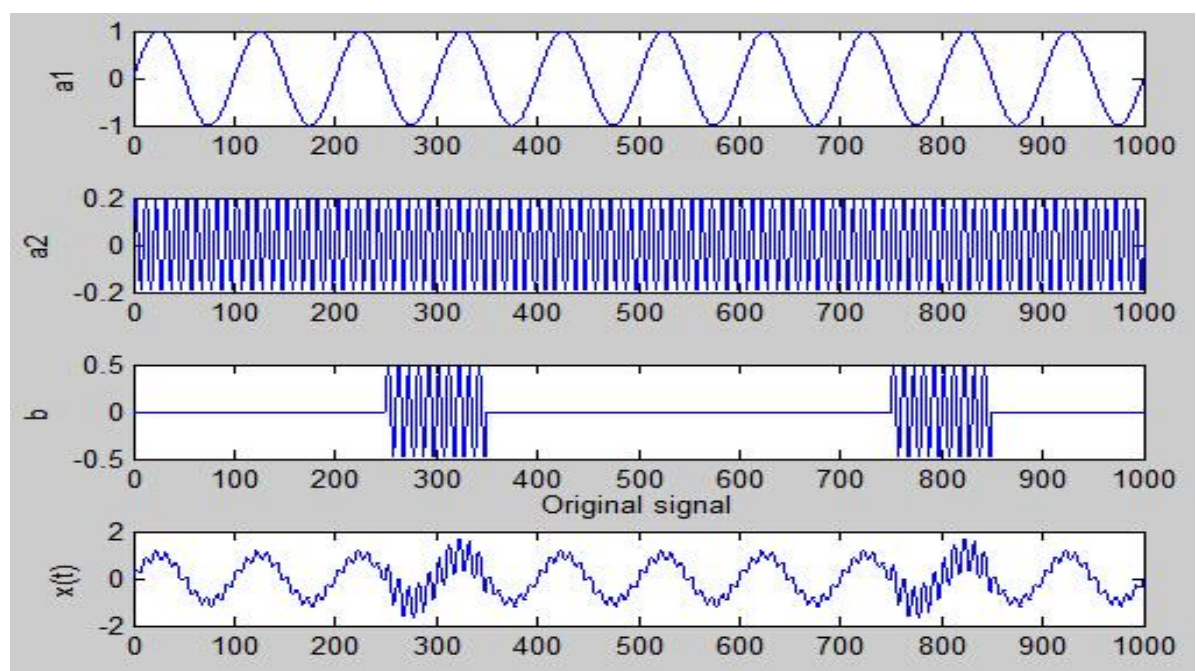

Fig.1 Mixed signals and its composition

$x(t)$ simulation of EEMD, among them, the use of the software is MATLAB(R2013a),results as shown in figure 2 .

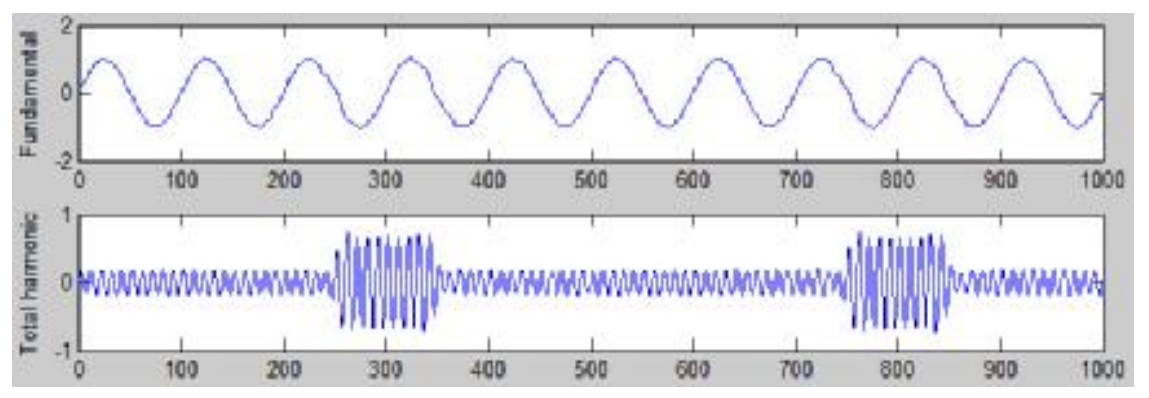

Fig. 2 Improvement EEMD simulation results

From the results of Figure 2, it can be seen that the improved EEMD algorithm can effectively find the fundamental wave in the superimposed signal, and make the difference can get the total harmonic and the effect is good.

\section{Application of Harmonic Detection and Control in Power System}

Added the Improved EEMD Algorithm Many experiments show that the above calculation 
method can lead to a large amount of residual harmonics in the fundamental wave, when the total harmonic distortion (THD)rate of the signal is large.So when THD larger, the following supplementary operation is performed on the above results (8):

Order $B_{m+1}(t)=f_{1}(t)$

$\mathrm{f}_{1}(\mathrm{t})$ as the original signal $\stackrel{\text { step 1),2),3)operation }}{\longrightarrow} \mathrm{b}_{1}(\mathrm{t})+\mathrm{B}_{1}(\mathrm{t})$;

$\mathrm{f}_{1}(\mathrm{t})-\mathrm{b}_{1}(\mathrm{t})$ as the original signal $\stackrel{\text { step 1),2),3) operation }}{\longrightarrow} \mathrm{b}_{2}(\mathrm{t})+\mathrm{B}_{2}(\mathrm{t})$;

1

$\mathrm{f}_{\mathrm{m}-1}(\mathrm{t})-\mathrm{b}_{\mathrm{m}-1}(\mathrm{t})$ as the original signal $\stackrel{\text { step 1),2),3) operation }}{\longrightarrow} \mathrm{b}_{\mathrm{m}}(\mathrm{t})+\mathrm{B}_{\mathrm{m}}(\mathrm{t})$;

$b_{1}(t), b_{2}(t), \ldots b_{m+1}(t)$, respectively, for the first two of the decomposed component of the IMF.

Take $\mathrm{m}=800 ; \mathrm{B}_{\mathrm{m}}(\mathrm{t})$ is the fundamental wave. $\mathrm{S}(\mathrm{t})-\mathrm{B}_{\mathrm{m}}(\mathrm{t})=\mathrm{R}_{\mathrm{S}}(\mathrm{t})$ is the total harmonic.

Simulate the Distortion Alternating Current and Carry Out the Simulation Experiment Use MATLAB (R2013a) to simulation output distortion alternating current. The model is shown in Figure 3.

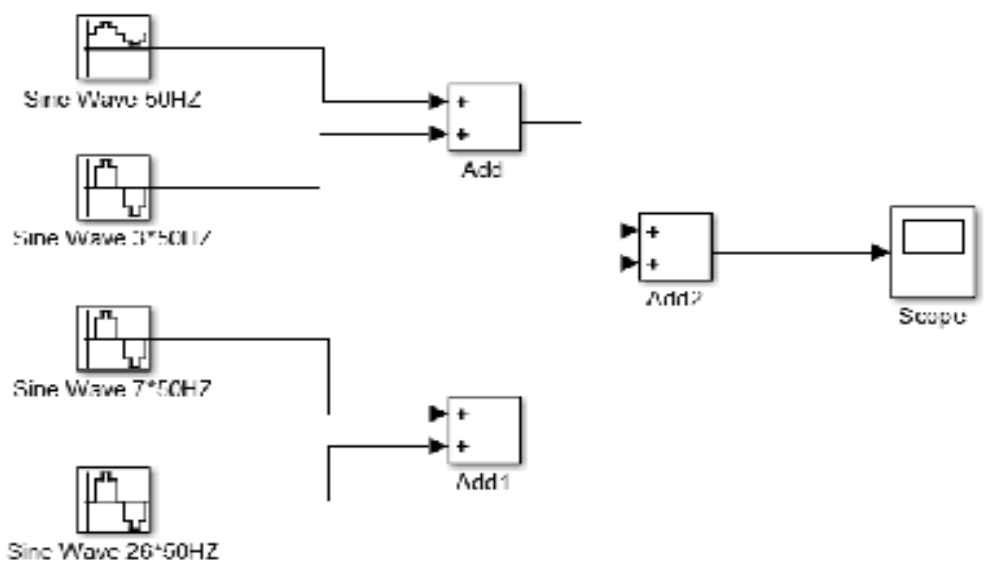

Fig.3 Distorted alternating current simulation model

In the model, 3 times, 7 times and 26 times of the harmonic are superimposed, including the fundamental, odd and even order, higher order harmonics, and added a 0.7 bias in the fundamental wave, used to simulate the DC component.All parameters is shown in Table 1:

\begin{tabular}{|c|c|c|c|c|c|}
\hline Sine Wave & Amplitude & bias & Frequency(rad/sec) & Phase(rad) & Sample Time \\
\hline $50 \mathrm{HZ}$ & 2 & 0.7 & $2 * \mathrm{pi} * 50$ & 0 & 0.001 \\
\hline $3 * 50 \mathrm{HZ}$ & 1 & 0 & $2 * \mathrm{pi} * 50 * 3$ & 90 & 0.001 \\
\hline $7 * 50 \mathrm{HZ}$ & 0.8 & 0 & $2 * \mathrm{pi} * 50 * 7$ & 180 & 0.001 \\
\hline $26 * 50 \mathrm{HZ}$ & 0.6 & 0 & $2 * \mathrm{pi} * 50 * 26$ & 270 & 0.001 \\
\hline
\end{tabular}

Table 1 Simulation input data

Output waveform distortion of the current model as shown in figure4. 


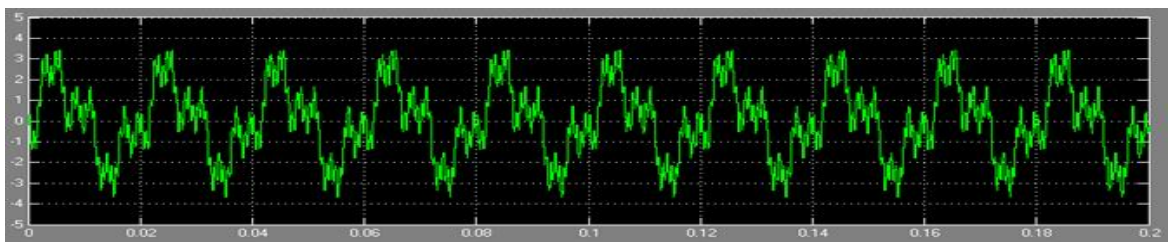

Fig.4 Simulated distortion current

Simulation of distorted current, results as shown in Figure 5.

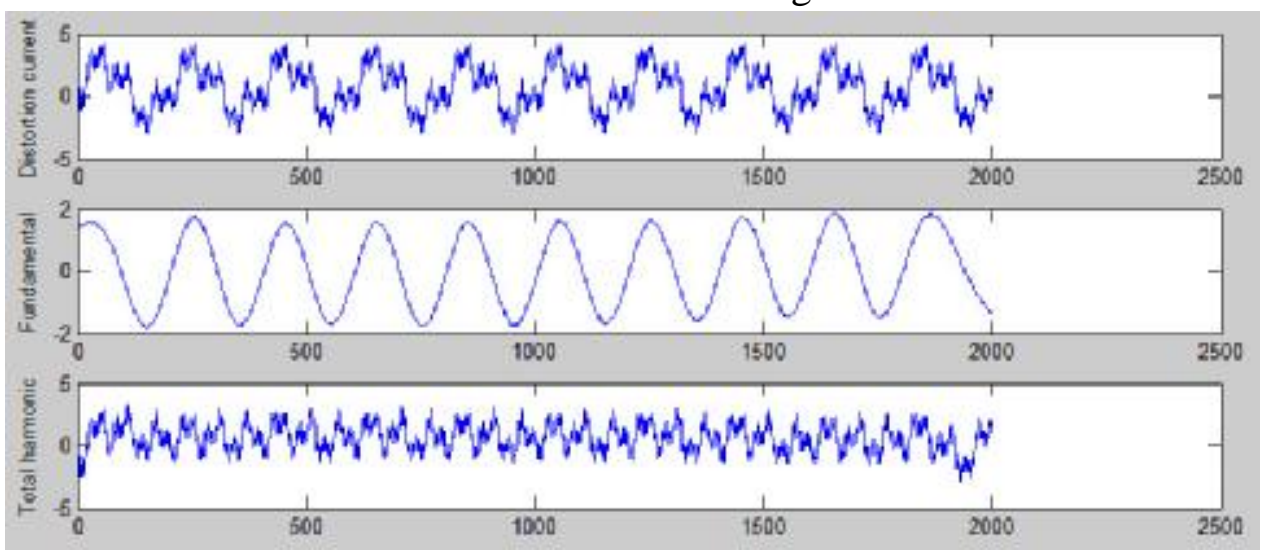

Fig.5 Primary distortion current, Fundamental current and Total harmonic current

From the simulation results, we can see that the improved EEMD algorithm can detect and separate the fundamental current and harmonic current, and the effect is good,as long as in the original current added in the total harmonic current are equal in size, in the opposite direction of supply current, the harmonic can be realized in governance.

\section{Conclusions}

The improved EEMD algorithm proposed in this paper can be used to filter the fundamental wave in the mixed wave. The simulation results show that the proposed method is suitable for power system harmonic detection and control. But the method is not perfect, and it needs to be further improved.

\section{Acknowledgement}

This research was financially supported by scientific research innovation projects of Shanghai municipal education commission (Grant No.13YZ140) and the key disciplines of Shanghai Municipal Education Commission of China (Grant No.J51901)

\section{References}

[1]Han B M,Bae B Y,Ovaska S J.Reference signal generator for active power filters using improved adaptive predictive filter[J].IEEE Transactions on Industrial Electronics,2005,52(2):576-584.

[2]Huang $\mathrm{N}$ E,Shen Z,Long S R,et al.The empirical mode decomposition and the Hilbert spectrum for nonlinear and non-stationary time series analysis,Proc. Roy.Soc.London,1998,454: 903-995.

[3]Huang $\mathrm{N}$ E,Shen Z,Long RS.A new view of nonlinear water waves-the Hilbert spectrum,Ann.Rev.Fluid Mech,1999,31: 417-457.

[4]YU Dejie,CHEN Junsheng,YANG Yu.Hilbert-Huang transform method for mechanical fault diagnosis [M].Beijing: Science Press,2007.

[5]Huang N E,Wu Z.A review on Hilbert - Huang transform: Method and its applications to geophysical studies,Advances in Adaptive Data Analysis 2009,1: 1-23. 
[6]Gai GH.The processing of rotor startup signals based on empirical mode decomposition[J].Mechanical Systems and Signal Processing,2006,20: 225-235.

[7]LEI Ya-guo.Machinery fault diagnosis based on improved Hilbert-Huang transform [ J].Chinese Journal of Mechanical Engineering,2011,47( 5) : 71-77.

[8]Wu Z H,Huang N E.Ensemble empirical mode decomposition: A noise assisted data analysis method [J].Advances in Adaptive Data Analysis,2009,1: 1-41.

[9]Yeh J R, Shieh J S.Complementary ensemble empirical mode decomposition: A noise enhanced data analysis method [J],Advances in Adaptive Data Analysis,2010,2 (2) : 135-156. 